\title{
Does Creatine Phosphokinase MB Predict Long-Term Cardiac Death or Atrial Fibrillation?
}

\author{
Karoline Korsholm Jeppesen*, Sara Schødt Riber, Lars Peter Riber \\ Department of Cardiac, Thoracic and Vascular Surgery, Odense University Hospital, Odense, Denmark \\ Email: ^Karoline.Kor sholm. Jeppesen@rsyd.dk
}

How to cite this paper: Jeppesen, K.K., Riber, S.S. and Riber, L.P. (2019) Does Creatine Phosphokinase MB Predict Long-Term Cardiac Death or Atrial Fibrillation? Open Journal of Thoracic Surgery, 9, 43-58. https://doi.org/10.4236/ojts.2019.94005

Received: November 16, 2019

Accepted: December 9, 2019

Published: December 12, 2019

Copyright $\odot 2019$ by author(s) and Scientific Research Publishing Inc. This work is licensed under the Creative Commons Attribution International License (CC BY 4.0).

http://creativecommons.org/licenses/by/4.0/

\section{cc (i) Open Access}

\begin{abstract}
Objectives: Exploring the long-term consequences of elevated postoperative creatine phosphokinase $\mathrm{MB}$ as a surrogate measure of cardiac tissue damage subsequent to coronary artery bypass graft surgery (CABG). Methods: Prospective cohort study including 414 patients subjected to solitary CABG at Odense University Hospital from September 30, 2007, and backwards in time. According to individual postoperative peak creatine phosphokinase MB, patients were assigned to five subgroups. Kaplan Meier survival analysis and Cox proportional-hazard regression evaluated the predictive value of creatine phosphokinase $\mathrm{MB}$ on late death from cardiac cause and diagnosis of paroxysmal or persistent atrial fibrillation within 10-years after CABG. Results: There was a general numeric decrease in time to cardiac death with increasing postoperative creatine phosphokinase MB (Log-Rank 0.285). Creatine phosphokinase $\mathrm{MB}<25$ was significantly associated with both shortened extracorporeal circulation time (HR 0.42; 95\% CI $0.30-0.57 ; p<0.001)$ and aortic cross-clamp time (HR 0.54; 95\% CI $0.40-0.73 ; p<0.001$ ) compared to creatine phosphokinase $\mathrm{MB} \geq 100$. No association between creatine phosphokinase $\mathrm{MB}$ and atrial fibrillation was identified. Postoperative atrial fibrillation occurred in 84 patients, whereof 21 later developed paroxysmal or persistent atrial fibrillation. There was a statistically significant 2.4-fold (HR 2.38; 95\% CI 1.37 - 4.14; $p=0.002$ ) increase in paroxysmal or persistent atrial fibrillation in patients with postoperative atrial fibrillation compared to patients in postoperative sinus rhythm. Conclusions: Postoperative creatine phosphokinase MB was not found predictive of late cardiac death or diagnosis of paroxysmal or persistent atrial fibrillation within 10 years after CABG. However, patients with postoperative atrial fibrillation had increased risk of later developing paroxysmal or persistent atrial fibrillation compared to patients in postoperative sinus rhythm.
\end{abstract}

\section{Keywords}

Coronary Artery Bypass Graft Surgery, Creatine Phosphokinase MB, Cardiac 
Mortality, Atrial Fibrillation

\section{Introduction}

Annually, around 1400 on pump coronary artery bypass graft surgeries (CABG) are performed in Denmark [1]. Data demonstrate a postoperative mortality of $1.3 \%$ within 30 days increasing to $3.0 \%$ within 12 months in elective cases [2].

\subsection{Creatine Phosphokinase MB}

Postoperative biomarker measurements serve to assess effect of revascularization procedures and potential surgery induced damage to cardiac tissue. The biomarker creatine phosphokinase MB (CK-MB) is routinely measured four hours after removal of the aortic cross-clamp and the next morning. Additional CK-MB measurements are performed if deemed necessary.

$\mathrm{CK}-\mathrm{MB}$ resides mainly in the striated musculature in cardiac tissue, where it catalyses cleavage of high-energy phosphate from adenosine triphosphate resulting in energy release. Cardiac muscle breakdown causes release of CK-MB to the blood circulation, hence, enabling detection of myocardial injury in a simple blood sample.

Myocardial infarction is defined as myocardial necrosis due to prolonged ischemia. However, myocardial injury with necrosis may be detected in conditions subsequent to non-ischemic myocardial damage including heart failure, myocarditis, arrhythmias, renal failure, pulmonary embolism and otherwise uncomplicated percutaneous or surgical interventions [3].

\subsection{Mortality}

Steuer et al. [4] found patients with CK-MB $\geq 61 \mu \mathrm{g} / \mathrm{l}$ after CABG were in increased risk of cardiac death within 30 days. This addresses the immediate clinical relevance of CK-MB more than long-term prognostic capability. Domanski et al. [5] documented a significant association between elevated CK-MB and increased cardiovascular mortality within 4 years after CABG. However, the study only included patients with diabetes mellitus, which impair extrapolation to a general CABG population. Costa et al. [6] showed elevated postoperative CK-MB to be significantly associated with late adverse outcome.

The duration of extracorporeal circulation time (ECC) and aortic cross-clamp time (CC) has been correlated with early postoperative adverse events but not long-term outcomes [7]. The stress of ECC and CC duration on cardiac tissue needs further quantification.

\subsection{Atrial Fibrillation}

Postoperative atrial fibrillation (POAF) occurs in around 35\% of patients in the immediate period after solitary CABG [8] [9]. POAF develops most frequently 
on the second to third postoperative day [8].

The mechanisms responsible for POAF are not fully understood. It appears that preoperative degenerative changes in the atrial myocardium combined with perioperative circumstances changing a number of electrophysiological parameters create a myocardium prone to supraventricular arrhythmias [9]. The only factor consistently associated with greater risk of POAF is age, presumably due to development of fibrotic tissue and vulnerability to electrophysiological changes [9] [10].

POAF is often self-limiting and spontaneously converts to sinus rhythm regardless of treatment [11]. However, POAF increases the risk of thromboembolic events [12] [13], why it is standard practice to convert POAF to sinus rhythm immediately.

The mechanism responsible for late onset POAF have yet to be determined, given it is not merely a reflection of a general increase in arrhythmia with age. As mentioned, CK-MB is a marker of myocardial ischemia, why elevated postoperative levels are an indicator of insufficient myocardial preservation, myocardial damage due to surgery performed or a result of insufficient coronary re-vascularisation. The potential of $\mathrm{CK}-\mathrm{MB}$ as a parameter in atrial fibrillation risk prediction algorithms need evaluation.

\subsection{Study Aims}

The primary objective is to investigate if peak creatine phosphokinase MB after uncomplicated solitary CABG predicts late cardiac death within 10-year postoperative follow-up. Further, the effect of extracorporeal circulation time and aortic cross-clamp time on creatine phosphokinase MB is investigated.

The secondary objective is to investigate if peak creatine phosphokinase $\mathrm{MB}$ or postoperative atrial fibrillation after uncomplicated solitary CABG are predictors of later diagnosis of paroxysmal or persistent atrial fibrillation.

\section{Patients and Methods}

\subsection{Study Design}

Prospective cohort study.

\subsection{Study Population}

The Western Denmark Heart Register supplied information regarding patients subjected to CABG at Odense University Hospital from January 1, 1999, to September 30, 2007.

Inclusion criteria were solitary CABG in patients discharged from the hospital within 14 days and still alive 30 days postoperative.

Exclusion criteria were indications of ischemia confer the World Health Organization International Classification of Diseases (ICD-10) [14] diagnostic code assigned to each patient upon hospital admission. Hence, exclusion of patients with unstable angina pectoris, acute myocardial infarction or patients subjected 
to acute percutaneous coronary intervention prior to CABG.

\subsection{Descriptive Variables}

Patients were assigned to one of five predetermined CK-MB intervals $(<25,25$ $49,50-74,75$ - 99 and $\geq 100$ ) according to individual peak postoperative CK-MB measurement. Including the 100 patients with most recent surgeries in each CK-MB interval from 30 September 2007 and backward in time.

The Western Denmark Heart Register furthermore supplied information on preoperative baseline patients characteristics, procedural and postoperative variables. Procedural variables considered in the study were central and peripheral anastomosis, ECC, CC and administration of blood, plasma, thrombocyte and colloid volume. Postoperative variables studied were CNS injury, sternal infection, reoperation for bleeding or ischemia, acute myocardial infarction, new Q-wave on ECG, EuroSCORE and admission time.

\subsection{Outcome Variables}

The Danish Cause of Death register supplied data, which was transformed into a dichotomous variable by categorising the primary cause of death as of non-cardiac or cardiac origin. E.g. heart failure, ischemic heart disease, myocardial infarction, arrhythmia et cetera.

The Danish National Patient register provided information on hospital contacts. Atrial fibrillation (AF) was defined as any diagnosis of paroxysmal or persistent atrial fibrillation more than 30 days after surgery. Diagnosis of atrial fibrillation within 30 days after surgery was considered postoperative atrial fibrillation (POAF).

Patients diagnosed with arrhythmia prior to $\mathrm{CABG}$ were excluded from analysis.

\subsection{Statistical Analysis}

Potential differences in binomial variables between CK-MB subgroups were tested with Pearson- $x^{2}$ coefficient or Fishers exact test. Significant test statistics were followed with calculation of $z$-score and associated $\mathrm{p}$-value for each subgroup. The alpha level was adjusted according to the Bonferroni correction for multiple comparisons.

Potential differences in ordinal variables between subgroups were assessed with Kruskal Wallis analysis. Significant test statistics were supplemented with post hoc pairwise Mann-Whitney test with Bonferroni correction.

Distribution of quantitative variables was tested with histogram, q-q plot and Shapiro-Wilk test. Equality of variance was explored using Levene's test.

Potential differences between subgroups in quantitative variables with normal distribution and equality of variance were disclosed by One-Way ANOVA analysis. Significant test statistics were followed with Scheffe post hoc test. Quantitative variables with normal distribution but inequality of variance were tested with Brown Forsythe and Welch test and post hoc Games-Howell test. 
Quantitative variables with a distribution other than normal were log transformed to normalise distribution to satisfy the One-Way ANOVA condition. If the log transformed distribution did not approximate normality Kruskal Wallis test was used. In case of significant test statistics, pairwise Mann-Whitney test with Bonferroni correction, was performed.

Kaplan Meier survival curves with time to event late cardiac death were drawn for each of the five subgroups. Differences in estimates between subgroups were tested with Log-Rank.

Variables found to be significantly different between subgroups were tested in regard to potential confounding. Each variable was tested using univariable Cox proportional-hazard regression with time to event late cardiac death and event $\mathrm{AF}$ as the dependent variables, respectively. Confounders were identified at a significance level of $5 \%$ ( $p$-value $<0.05$ ). The proportionality assumption for the Cox models was tested with Schoenfeld residuals.

Univariable Cox proportional-hazard regression was subsequently used to evaluate the relationship between CK-MB and both ECC and CC.

A multivariable Cox proportional-hazard regression model was produced for late cardiac death and AF, respectively. Potential confounders identified in the previous analysis were adjusted for in the two models.

Cumulative hazard functions assessed the correlation between POAF and AF. Differences in baseline characteristics between the POAF patients and patients in postoperative sinus rhythm were considered with Pearson- $x^{2}$ coefficient or Fishers exact test for categorical variables and independent sample T-test for normal distributed quantitative variables. Variables with a distribution other than normal were assessed with non-parametric Mann-Whitney test. A multivariable Cox proportional-hazard regression model with time to event AF adjusted for possible confounders was produced.

Cox proportional-hazard regression was subsequently used for evaluating the risk of all-cause death and cardiac death following POAF and AF.

The proportionality assumptions for the overall multivariable Cox models were tested with global Schoenfeld residuals test. The goodness-of-fit of the models were estimated by Martingale residuals and associated Cox-Snell residuals.

Values with a two-sided alpha level of $p<0.05$ were considered statistically significant.

IBM SPSS 25.0 and STATA 15 statistical software were used for statistical analysis.

\subsection{Interobserver Analysis}

An interobserver analysis was performed to evaluate the validity of the information from The Western Denmark Heart Register. From each CK-MB subgroup, $10 \%$ of patients were chosen at random. Two researchers (KKJ and LPR) independently reviewed patient records. Discrepancies were resolved by consensus. The information from The Western Denmark Heart register was compared to 
the information obtained from patient records by calculating Cohen's $\kappa$ -coefficient, variable sensitivity and specificity.

\subsection{Approvals}

The Danish Data Protection Agency and the Danish Patient Safety Authority approved the study. The Regional Committees on Health Research Ethics for Southern Denmark waived consent requirements.

\section{Results}

The study evaluated 414 patients. Baseline characteristics for the five CK-MB subgroups were balanced with respect to preoperative baseline characteristics (Table 1).

\subsection{CK-MB and Late Cardiac Death}

In the 10-year follow-up period 105 of 414 patients died, whereof 33 deaths were of cardiac origin. Three patients died from unknown causes.

The Kaplan Meier survival estimates of time to late cardiac death generally decreased with an increasing level of CK-MB especially pronounced after 5 years in subgroups with CK-MB $>75 \mu \mathrm{g} / \mathrm{l}$ (Figure 1). However, Log-Rank test did not identify any statistically significant difference between subgroups.

Neither univariable nor multivariable Cox proportional-hazard regression analysis identified any statistical significant association between postoperative CK-MB level and late cardiac death (Table 2).

Subsequent analysis showed a statistically significant decrease in both ECC and CC in subgroup CK-MB $<25$ compared to subgroup CK-MB $\geq 100$ (Table 3).

\section{2. $\mathrm{CK}-\mathrm{MB}$ and $\mathrm{AF}$}

AF occurred in 54 out of 414 patients, within 10 years. No association between postoperative $\mathrm{CK}-\mathrm{MB}$ level and time to AF was identified in univariable or multivariable Cox proportional-hazard analysis (Table 4).

No differences between CK-MB subgroups regarding postoperative CNS injury, sternal infection or reoperation for bleeding were found. An increased postoperative CK-MB level was associated with a significantly higher risk of postoperative acute myocardial infarction, new Q-wave, higher EuroSCORE, reoperation due to ischemia and prolonged intensive care unit admission time.

\subsection{POAF and AF}

POAF occurred in 84 out of 414 patients, whereof 21 later developed AF. Among the 330 patients in postoperative sinus rhythm only 33 patients later developed AF (Figure 2).

Beta-blocker treatment was significantly more frequent in patients with postoperative sinus rhythm (76\%) compared to patients with POAF (64\%; p-value = 
Table 1. Baseline characteristics.

\begin{tabular}{|c|c|c|c|c|c|c|}
\hline & \multicolumn{6}{|c|}{ Creatine phosphokinase MB subgroup } \\
\hline & $\mathrm{n}$ & $<25[\mu \mathrm{g} / 1]$ & $25-49[\mu \mathrm{g} / \mathrm{l}]$ & $50-74[\mu \mathrm{g} / \mathrm{l}]$ & $75-99[\mu \mathrm{g} / \mathrm{l}]$ & $\geq 100[\mu \mathrm{g} / \mathrm{l}]$ \\
\hline & 414 & $(\mathrm{n}=92)$ & $(\mathrm{n}=99)$ & $(\mathrm{n}=77)$ & $(\mathrm{n}=67)$ & $(\mathrm{n}=79)$ \\
\hline \multicolumn{7}{|l|}{ Demographics } \\
\hline Age_years (IQR) & 414 & $63(45-83)$ & $64(39-82)$ & $65(38-84)$ & $66(44-80)$ & $63(37-82)$ \\
\hline Males_number (\%) & 414 & $77(84)$ & $92(93)$ & $55(72)$ & $45(67)$ & $60(76)$ \\
\hline Body-mass-index_kg/m² (SD) & 411 & $28(4)$ & $29(4)$ & $28(6)$ & $27(4)$ & $27(4)$ \\
\hline \multicolumn{7}{|l|}{ Preoperative } \\
\hline Smoking_number (\%) & 403 & & & & & \\
\hline Never & & $11(12)$ & $27(28)$ & $23(31)$ & $19(28)$ & $21(29)$ \\
\hline Previous & & $50(55)$ & $48(50)$ & $33(44)$ & $34(51)$ & $31(43)$ \\
\hline Active & & $30(33)$ & $22(23)$ & $19(25)$ & $14(21)$ & $21(29)$ \\
\hline Ejection fraction_\% (SD) & 413 & $51(9)$ & $51(11)$ & $51(10)$ & $54(12)$ & $53(13)$ \\
\hline S-creatinin_mmol/l (IQR) & 412 & $96(68-1002)$ & $97(25-800)$ & $96(62-192)$ & $98(69-201)$ & $97(70-171)$ \\
\hline \multicolumn{7}{|l|}{ Coexisting_number (\%) } \\
\hline Ischemic heart disease & 398 & $33(36)$ & $47(48)$ & $39(51)$ & $34(55)$ & $33(47)$ \\
\hline Diabetes mellitus & 414 & $18(20)$ & $18(18)$ & $14(18)$ & $8(12)$ & $6(8)$ \\
\hline Chronic obstructive pulmonary disease & 404 & $6(7)$ & $1(1)$ & $5(7)$ & $5(8)$ & $5(7)$ \\
\hline Peripheral artery disease & 414 & $15(16)$ & $9(9)$ & $8(10)$ & $3(5)$ & $12(15)$ \\
\hline Previous cardiac surgery & 414 & $0(0)$ & $6(6)$ & $4(5)$ & $6(9)$ & $8(10)$ \\
\hline Previous percutaneous coronary intervention & 414 & $17(19)$ & $20(20)$ & $10(13)$ & $9(13)$ & $10(13)$ \\
\hline Previous myocardial infarction & 407 & $37(40)$ & $51(52)$ & $35(46)$ & $29(43)$ & $44(60)$ \\
\hline \multicolumn{7}{|l|}{ Medication_number (\%) } \\
\hline Lipid & 412 & $80(87)$ & $89(90)$ & $62(81)$ & $41(62)$ & $49(63)$ \\
\hline Hypertensive & 411 & $49(53)$ & $68(69)$ & $43(56)$ & $30(46)$ & $31(40)$ \\
\hline Beta-blocker & 413 & $62(67)$ & $73(74)$ & $59(77)$ & $49(73)$ & $60(77)$ \\
\hline Calcium-antagonist & 413 & $29(32)$ & $39(39)$ & $29(38)$ & $27(40)$ & $19(24)$ \\
\hline Angiotensin converting enzyme inhibitor & 414 & $51(55)$ & $45(46)$ & $32(42)$ & $22(33)$ & $21(27)$ \\
\hline
\end{tabular}

Normally distributed variables are noted as mean with standard deviation, others as median with interquartile range.

0.035). There was no correlation between beta-blocker treatment and later diagnosis of AF.

Age and EuroSCORE were significantly different between the POAF patients and patients in postoperative sinus rhythm, while also being independently associated with late AF. However, multivariable Cox proportional-hazard analysis with time to event AF remained significantly associated with POAF when adjusting for age and EuroSCORE (Table 5). 


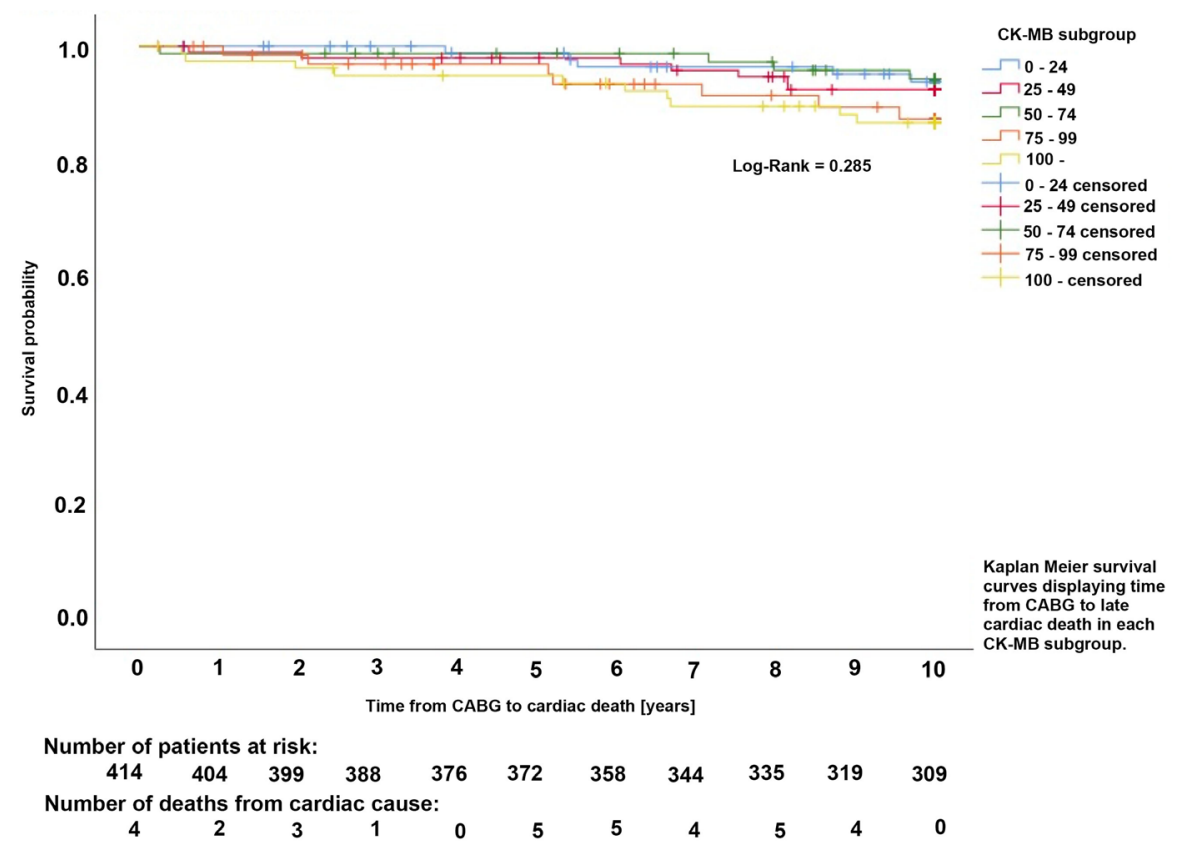

Figure 1. Kaplan Meier survival analysis.

Table 2. Risk of late cardiac death.

\begin{tabular}{|c|c|c|c|c|c|c|c|}
\hline \multirow[b]{2}{*}{ Variables } & & \multirow[b]{2}{*}{$\mathrm{n}$} & \multirow{2}{*}{$\begin{array}{c}\text { Late cardiac } \\
\text { death }(\%)\end{array}$} & \multicolumn{2}{|c|}{ Univariable } & \multicolumn{2}{|c|}{ Multivariable } \\
\hline & & & & HR $(95 \%$ CI $)$ & p-value & HR (95\% CI) & p-value \\
\hline \multirow{5}{*}{$\mathrm{CK}-\mathrm{MB}[\mu \mathrm{g} / \mathrm{l}]$} & $<25$ (ref.) & 91 & $5(5.5)$ & 1 & 0.319 & 1 & 0.763 \\
\hline & $25-49$ & 97 & $7(7.2)$ & $1.27(0.40-3.99)$ & 0.688 & $0.98(0.30-3.15)$ & 0.917 \\
\hline & $50-74$ & 77 & $4(5.2)$ & $0.92(0.25-3.42)$ & 0.900 & $0.48(0.12-1.92)$ & 0.301 \\
\hline & $75-99$ & 67 & $7(10.4)$ & $2.15(0.68-6.78)$ & 0.191 & $1.07(0.30-3.80)$ & 0.919 \\
\hline & $\geq 100$ & 79 & $10(12.7)$ & $2.32(0.79-6.78)$ & 0.125 & $0.82(0.25-2.70)$ & 0.742 \\
\hline \multirow{3}{*}{$\begin{array}{l}\text { Left ventricular ejection } \\
\text { fraction }[\%]\end{array}$} & $>50$ (ref.) & 175 & $8(4.7)$ & 1 & 0.072 & 1 & 0.581 \\
\hline & $31-50$ & 222 & $23(10.4)$ & $2.48(1.11-5.55)$ & 0.027 & $1.53(0.62-3.77)$ & 0.363 \\
\hline & $<30$ & 16 & $2(12.5)$ & $3.05(0.65-14.37)$ & 0.158 & $0.96(0.17-5.47)$ & 0.960 \\
\hline \multirow{2}{*}{ Previous cardiac surgery } & 0 & 387 & $28(7.2)$ & 1 & & 1 & \\
\hline & $\geq 1$ & 24 & $5(20.8)$ & $3.06(1.18-7.92)$ & 0.021 & $2.05(0.77-5.43)$ & 0.151 \\
\hline $\begin{array}{c}\text { Extracorporeal } \\
\text { circulation time [min.] }\end{array}$ & & 413 & & $1.01(1.00-1.02)$ & 0.009 & $1.01(1.00-1.02)$ & 0.018 \\
\hline EuroSCORE & & 414 & & $1.19(1.10-1.30)$ & $<0.001$ & $1.20(1.07-1.35)$ & 0.002 \\
\hline
\end{tabular}

Risk of late cardiac death evaluated with univariable and multivariable Cox analysis. Multivariable Cox analysis is adjusted for potential confounders: left ventricular ejection fraction, previous cardiac surgery, extracorporeal circulation time and EuroSCORE. ECC and EuroSCORE remained significantly associated with late cardiac death. Abbreviations: CK-MB: creatine phosphokinase MB, HR: hazard ratio.

\subsection{AF and late Cardiac Death}

Cardiac death was more frequent in POAF and AF patients compared to patients in sinus rhythm (Table 6).

Median admission time of patients in the POAF group was 175 hours (95\% CI 178 - 200), which was significantly longer compared to 159 hours (95\% CI 160 - 
Table 3. Risk of prolonged extracorporeal circulation time and aortic cross-clamp time.

\begin{tabular}{|c|c|c|c|c|}
\hline Variable & $\mathrm{CK}-\mathrm{MB}$ & $\mathrm{n}$ & HR $(95 \%$ CI $)$ & p-value \\
\hline \multirow{5}{*}{ Extracorporeal circulation time [min.] } & $<25$ & 92 & $0.42(0.30-0.57)$ & $<0.001$ \\
\hline & $25-49$ & 99 & $0.80(0.59-1.07)$ & 0.135 \\
\hline & $50-74$ & 77 & $1.10(0.80-1.50)$ & 0.552 \\
\hline & $75-99$ & 67 & $1.17(0.84-1.63)$ & 0.355 \\
\hline & $\geq 100$ (ref.) & 78 & 1 & \\
\hline \multirow{5}{*}{ Aortic cross - clamp time [min.] } & $<25$ & 92 & $0.54(0.40-0.73)$ & $<0.001$ \\
\hline & $25-49$ & 98 & $0.87(0.64-1.18)$ & 0.361 \\
\hline & $50-74$ & 76 & $1.23(0.89-1.70)$ & 0.215 \\
\hline & $75-99$ & 65 & $1.18(0.84-1.65)$ & 0.333 \\
\hline & $\geq 100$ (ref.) & 73 & 1 & \\
\hline
\end{tabular}

Effect of extracorporeal circulation time and aortic cross-clamp time on CK-MB. Abbreviations: CK-MB: creatine phosphokinase MB, HR: hazard ratio.

Table 4. Risk of later diagnosis of paroxysmal or persistent atrial fibrillation.

\begin{tabular}{|c|c|c|c|c|c|c|c|}
\hline \multirow[b]{2}{*}{ Variables } & & \multirow[b]{2}{*}{$\mathrm{n}$} & \multirow{2}{*}{$\mathrm{AF}(\%)$} & \multicolumn{2}{|c|}{ Univariable } & \multicolumn{2}{|c|}{ Multivariable } \\
\hline & & & & HR (95\% CI) & $\mathrm{p}$ - value & HR (95\% CI) & $\mathrm{p}$ - value \\
\hline \multirow{5}{*}{$\mathrm{CK}-\mathrm{MB}[\mu \mathrm{g} / \mathrm{l}]$} & $<25$ (ref.) & 92 & $16(17.4)$ & 1 & 0.561 & 1 & 0.324 \\
\hline & $25-49$ & 99 & $10(10.1)$ & $0.56(0.25-1.22)$ & 0.145 & $0.58(0.26-1.29)$ & 0.183 \\
\hline & $50-74$ & 77 & $11(14.3)$ & $0.81(0.37-1.74)$ & 0.583 & $0.72(0.33-1.57)$ & 0.411 \\
\hline & $75-99$ & 67 & $9(13.4)$ & $0.76(0.33-1.71)$ & 0.500 & $0.72(0.32-1.63)$ & 0.432 \\
\hline & $\geq 100$ & 79 & $8(10.1)$ & $0.56(0.24-1.30)$ & 0.178 & $0.40(0.17-0.97)$ & 0.042 \\
\hline Thrombocytevolume[ml] & & 413 & & $1.00(1.00-1.00)$ & 0.013 & $1.00(1.00-1.00)$ & 0.058 \\
\hline EuroSCORE & & 414 & & $1.12(1.03-1.21)$ & 0.005 & $1.12(1.03-1.21)$ & 0.010 \\
\hline
\end{tabular}

Risk of later diagnosis of AF evaluated with univariable and multivariable Cox analysis. Multivariable Cox analysis is adjusted for potential confounders: thrombocytevolume and EuroSCORE. Abbreviations: AF: paroxysmal or persistent atrial fibrillation, CK-MB: creatine phosphokinase MB, HR: hazard ratio.

168; $\mathrm{p}<0.001)$ in patients with sinus rhythm. Median intensive care unit admission time in the POAF group was 35 hours (95\% CI 38 - 47) compared to 34.5 hours (95\% CI $34-40 ; p=0.008$ ) for patients in postoperative sinus rhythm. Mean EuroSCORE in POAF patients were 5 (95\% CI 4.2 - 5.6), while 4 (95\% CI $3.7-4.3 ; \mathrm{p}=0.013)$ in patients with sinus rhythm.

\subsection{Interobserver Analysis}

The interobserver analysis showed a general underreporting in The Western Denmark Heart Register. The underreporting of comorbidities was moderate with Cohens $\kappa$-coefficient ranging from 0.53 in reporting of chronic obstructive pulmonary disease to 1.0 for diabetes mellitus. Notification regarding medicinal usage was varying from unreliability of hypertensive treatment $(\kappa=$ $0.05)$ to the well performing beta-blocker treatment $(\kappa=0.79)$ and ACE inhibitor treatment $(\kappa=0.80)$. Underreporting also applied for postoperative variables such as CNS injury, sternal infection and new Q-wave on ECG. 


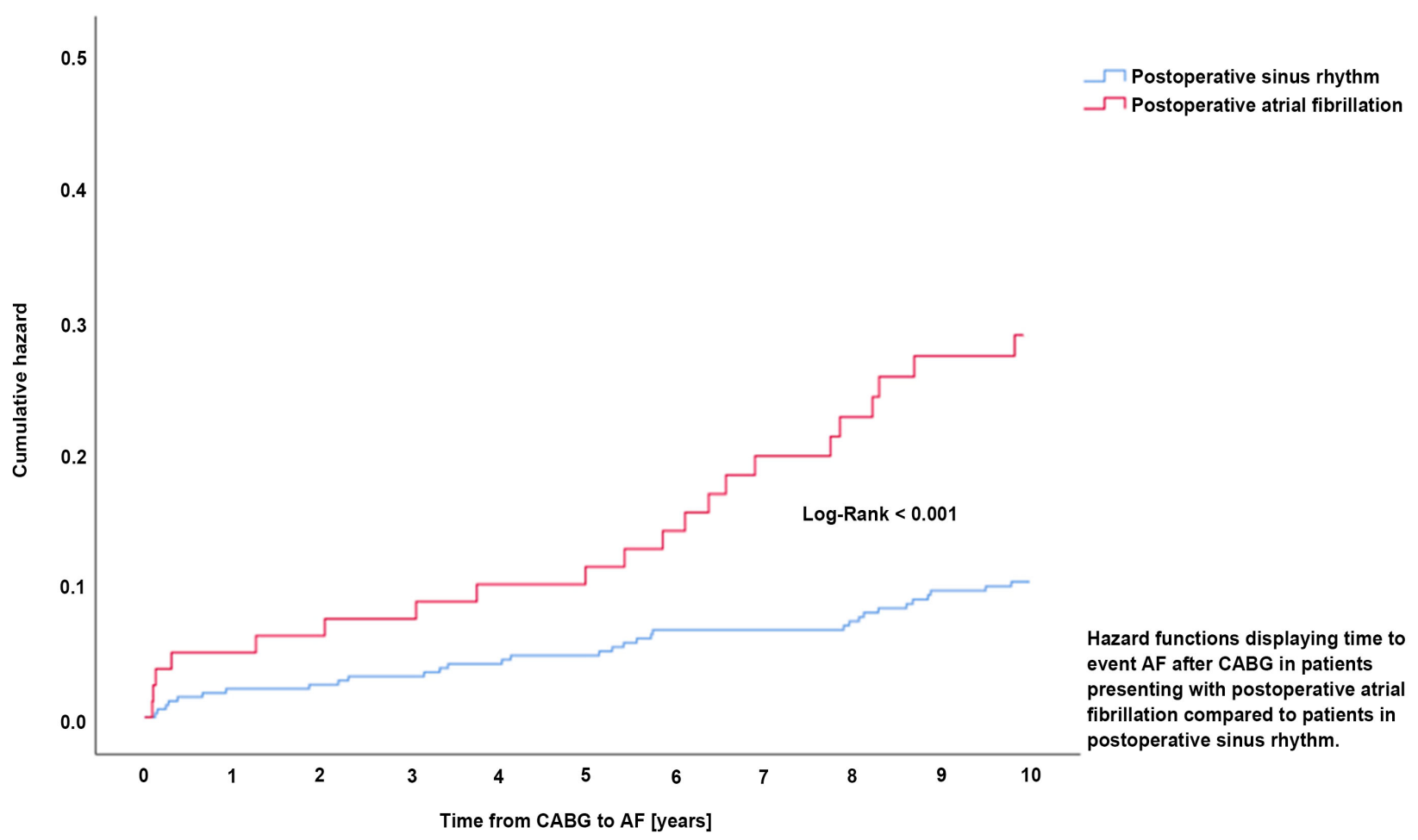

Figure 2. Hazard functions for time to paroxysmal or persistent atrial fibrillation (AF).

Table 5. Risk of later diagnosis of paroxysmal or persistent atrial fibrillation confer postoperative rhythm.

\begin{tabular}{ccccccc}
\hline & & \multicolumn{2}{c}{ Univariable } & \multicolumn{2}{c}{ Multivariable } \\
\hline Variable & $\mathrm{n}$ & $\mathrm{AF}(\%)$ & HR $(95 \% \mathrm{CI})$ & $\mathrm{p}$-value & HR $(95 \% \mathrm{CI})$ & p-value \\
\hline Sinus rhythm & 330 & $33(10.0)$ & 1 & & 1 & \\
POAF & 84 & $21(25.0)$ & $2.73(1.58-4.72)$ & $<0.001$ & $2.38(1.37-4.14)$ & 0.002 \\
Age[years] & 414 & & $1.05(1.02-1.09)$ & 0.002 & $1.03(0.99-1.07)$ & 0.095 \\
EuroSCORE & 414 & & $1.12(1.03-1.21)$ & 0.005 & $1.05(0.95-1.16)$ & 0.316 \\
\hline
\end{tabular}

Risk of later diagnosis of AF evaluated with univariable and multivariable Cox analysis. Multivariable Cox analysis is adjusted for potential confounders: age and EuroSCORE. Abbreviations: AF: paroxysmal or persistent atrial fibrillation, HR: hazard ratio, POAF: postoperative atrial fibrillation.

Table 6. Risk of late cardiac death in patients with POAF or AF compared to patients in sinus rhythm.

\begin{tabular}{ccccccc}
\hline \multirow{2}{*}{$\begin{array}{c}\text { Cardiac } \\
\text { rhythm }\end{array}$} & $\begin{array}{c}\text { Cause of } \\
\text { death }\end{array}$ & n (\%) & HR $(95 \% \mathrm{CI})$ & p-value & HR (95\% CI) & p-value \\
\cline { 5 - 7 } & & & & \multicolumn{2}{c}{ Univariable } & \multicolumn{2}{c}{ Multivariable } \\
\hline POAF $(\mathrm{n}=84)$ & All - cause & $28(33.3)$ & $1.51(0.98-2.32)$ & 0.064 & $1.23(0.79-1.90)$ & 0.354 \\
& Cardiac & $9(10.7)$ & $1.56(0.73-3.36)$ & 0.255 & $1.18(0.55-2.56)$ & 0.674 \\
& All - cause & $19(35.2)$ & $1.54(0.94-2.53)$ & 0.088 & $1.26(0.76-2.07)$ & 0.374 \\
AF (n=54) & Cardiac & $6(11.1)$ & $1.55(0.64-3.75)$ & 0.334 & $1.17(0.48-2.86)$ & 0.725 \\
\hline
\end{tabular}

Risk of late all-cause and cardiac death in patients with POAF or AF compared to patients in sinus rhythm, evaluated with univariable and multivariable Cox analysis. Multivariable Cox analysis is adjusted for age as a continuous variable. Abbreviations: AF: paroxysmal or persistent atrial fibrillation, HR: hazard ratio, POAF: postoperative atrial fibrillation. 
The variables ventricular dysfunction, renal function and POAF performed well with the latter having a Cohens $\kappa$-coefficient of 0.80 , sensitivity of $72.7 \%$ and specificity of $100 \%$.

Two patient records reported CABG to be acute and one reported a preoperative AF diagnosis, why they were excluded from analysis confer exclusion criteria.

\section{Discussion}

\subsection{CK-MB and Late Cardiac Death}

There was a numeric increased cardiac mortality among higher CK-MB subgroups, progressively pronounced beyond 5 years postoperative. However, the result must be interpreted with caution. As time progress after the CABG procedure, there is a growing risk of residual confounding influencing the result. Furthermore, a higher postoperative CK-MB level was significantly associated with an increased EuroSCORE, which indicates a more vulnerable cardiac risk profile in these patients. Hence, a shorter lifespan due to co-morbidities is to some extent expected in these patients.

Left ventricular ejection fraction, previous cardiac surgery, ECC and EuroSCORE were found to be significantly different between CK-MB subgroups and independently associated with late cardiac death. Presumably mirroring different steps in the cascade of mechanisms. Previous cardiac surgery might result in vulnerability of the myocardium. A high EuroSCORE indicates reduced resistance due to comorbidities, while decreased ejection fraction reflects declining cardiac function, which complicates sufficient cardioplegia and reperfusion of the myocardium. Hence, resulting in prolonged ECC and CC. Further, inadequate cardioplegia and thus suboptimal preservation of the myocardium during surgery results in increased CK-MB release.

\section{2. $\mathrm{CK}-\mathrm{MB}$ and $\mathrm{AF}$}

The study did surprisingly not identify any association between CK-MB and late AF. As elevated CK-MB correlated well with impaired cardiac function and arguably insufficient cardioplegia of the myocardium, then CK-MB would theoretically have been associated with increased risk of arrhythmia [15]. This finding suggests that development of AF is less dependent on the invasiveness of surgery and more so on pre-existing pathological substrate.

\subsection{POAF and AF}

The 2.4-fold increase in later diagnosis of AF in patients with POAF compared with patients in postoperative sinus rhythm could likewise indicate existence of pathological substrate in atrial myocardium that enables atrial arrhythmia. Thus leaving the myocardium prone for later development of AF regardless of immediate conversion to sinus rhythm.

The higher frequency of beta-blocker treatment among patients in postoperative 
sinus rhythm compared to patients with POAF is presumably a manifestation of the well known antiarrhythmic property of beta-blocker therapy [16].

The association between POAF and AF remained significant after adjusting for age. Furthermore, the hazard function for patients experiencing POAF increases at a higher rate from around 5 years postoperative compared to that of patients in postoperative sinus rhythm. If age were the main cause of AF, one would expect a more constant increase in AF with age.

These findings are consistent with the findings of Ahlsson et al. [17], who showed POAF to be associated with late AF and speculated if heart surgery could be viewed as a 'stress test' that unmasks a predisposition for AF. Melduni et al. [18] shared the hypothesis of a predisposing pathological substrate and showed that likelihood of recurrence of $\mathrm{AF}$ was coupled to the extent of underlying disease quantified by left ventricular diastolic dysfunction.

Although non-significant, patients with POAF were more likely to die from cardiac cause within 10 years after CABG compared to patients in sinus rhythm. This correlates well with previously found increased all-cause mortality due to POAF in large cohort studies [19] [20]. Ahlsson et al. [17] and Schwann et al. [21] found POAF to be correlated with increased cardiovascular mortality. Melduni et al. [18] did not find POAF significantly associated with all-cause mortality but did however find AF to be.

Therefore, the deficient significance regarding this matter in the present study is most likely due to underpower. However, accumulation of events in POAF patients could merely be a surrogate measure of the significantly higher EuroSCORE in POAF patients.

A number of other postoperative characteristics were considered. POAF patients were admitted significantly longer to both the regular hospital ward and intensive care unit. Furthermore, there was a higher incidence of postoperative CNS injury in POAF patients. POAF has previously been proved to increase the length of hospital stay in CABG patients [22] [23] and accumulate costs [24]. POAF prophylactic regimes have shown to significantly reduce risk of POAF and length of stay [25].

\subsection{Limitations and Strengths}

Data was collected prospectively. It is the first study to consider the long-term predictive capability of CK-MB on the risk of death confined to cardiac cause.

Knowledge of an association between POAF and AF is sparse, why this study reveals new data.

The cohort originates from a single-centre, which impair the external validity. Though it is noted, that the CABG procedure is relatively standardized. Therefore, extrapolation of the results might be reasonable.

\subsection{Data}

Missing register data were to a large extent acquired from patient files, reaching a low percentage of missing values. Register data are subjected to interobserva- 
tional variation.

\subsection{Population}

The requirement of including more patients to increase power and prevent type 2 errors was weighed against the resulting higher risk of residual confounding if patients from earlier surgical years were included in the study. If so, prompting a higher risk of confounding from differences in patient demographics and procedural factors.

\subsection{Descriptive Variables}

Postoperative CK-MB measurement is a standardized procedure and blood sample analyses were performed at the same laboratory for all patients. In addition, it has to be taken into consideration that even though CK-MB is considered specific to cardiac tissue, some amount of the CK-MB is produced in the brain tissue and striated skeletal muscle. As mentioned, elevated CK-MB can also be measured in other clinical conditions [3].

\subsection{Outcome Variables}

The Western Denmark Heart Register does not distinguish between atrial flutter and atrial fibrillation and the occurrence is only registered during hospital admission. However, a study by Zebis et al. [26] showed no occurrences of atrial flutter after CABG in a similar population and only a risk of $1 \%$ in developing atrial fibrillation after hospital discharge.

Munkholm et al. [27] evaluated the POAF variable in The Western Denmark Heart Register and calculated variable sensitivity 75.2\% (95\% CI 72.2 - 78.7), specificity $90.9 \%$ (95\% CI 88.8 - 92.6) and concluded that the POAF variable was reliable to be used in epidemiological studies. These findings correspond well with the results from the interobserver analysis.

The Danish Cause of Death Register was fully updated, why all patients had a complete 10-year followed-up. All patients were accounted for in The National Patient Register.

\section{Conclusions}

No association between elevated creatine phosphokinase MB level after solitary CABG and late cardiac death within 10 years was evident. However, creatine phosphokinase $\mathrm{MB}$ level $\geq 100$ was significantly associated with both prolonged extracorporeal circulation time and aortic cross-clamp time compared to a creatine phosphokinase MB level $<25$.

Creatine phosphokinase MB was not associated with a later diagnosis of paroxysmal or persistent atrial fibrillation within 10 years after solitary CABG. However, patients with postoperative atrial fibrillation presented with a statistically significant 2.4 -fold increase in later diagnosis of paroxysmal or persistent atrial fibrillation within 10 years postoperatively. 
Long-term follow-up in patients, who experience postoperative atrial fibrillation, may be beneficial to avoid later complications.

\section{Funding}

None declared.

\section{Conflicts of Interest}

The authors declare no conflicts of interest regarding the publication of this paper.

\section{References}

[1] Danish Health Authority (2017) Surveillance and Diagnostics in the Cardiac Field.

[2] Özcan, C.V.J., Davidsen, M. and Gislason, G. (2016) Annual Report: Western Denmark Heart Register 2016.

[3] Thygesen, K., Alpert, J.S., Jaffe, A.S., Simoons, M.L., Chaitman, B.R. and White, H.D. (2012) Third Universal Definition of Myocardial Infarction. Global Heart, 7, 275-295. https://doi.org/10.1016/j.gheart.2012.08.001

[4] Steuer, J., Horte, L.G., Lindahl, B. and Stahle, E. (2002) Impact of Perioperative Myocardial Injury on Early and Long-Term Outcome after Coronary Artery Bypass Grafting. European Heart Journal, 23, 1219-1227. https://doi.org/10.1053/euhj.2002.3171

[5] Domanski, M., Farkouh, M.E., Zak, V., French, J., Alexander, J.H., Bochenek, A., et al. (2016) Relation of Post-Coronary Artery Bypass Graft Creatine Kinase-MB Elevations and New Q Waves with Long-Term Cardiovascular Death in Patients with Diabetes Mellitus and Multivessel Coronary Artery Disease. American Journal of Cardiology, 118, 1655-1660. https://doi.org/10.1016/j.amjcard.2016.08.041

[6] Costa, M.A., Carere, R.G., Lichtenstein, S.V., Foley, D.P., de Valk, V., Lindenboom, W., et al. (2001) Incidence, Predictors, and Significance of Abnormal Cardiac Enzyme Rise in Patients Treated with Bypass Surgery in the Arterial Revascularization Therapies Study (ARTS). Circulation, 104, 2689-2693. https://doi.org/10.1161/hc4701.099789

[7] Salis, S., Mazzanti, V.V., Merli, G., Salvi, L., Tedesco, C.C., Veglia, F., et al. (2008) Cardiopulmonary Bypass Duration Is an Independent Predictor of Morbidity and Mortality after Cardiac Surgery. Journal of Cardiothoracic and Vascular Anesthesia, 22, 814-822. https://doi.org/10.1053/j.jvca.2008.08.004

[8] Maisel, W.H., Rawn, J.D. and Stevenson, W.G. (2001) Atrial Fibrillation after Cardiac Surgery. Annals of Internal Medicine, 135, 1061-1073. https://doi.org/10.7326/0003-4819-135-12-200112180-00010

[9] Maesen, B., Nijs, J., Maessen, J., Allessie, M. and Schotten, U. (2012) Post-Operative Atrial Fibrillation: A Maze of Mechanisms. Europace, European Pacing, Arrhythmias, and Cardiac Electrophysiology, 14, 159-174. https://doi.org/10.1093/europace/eur208

[10] Goette, A., Juenemann, G., Peters, B., Klein, H.U., Roessner, A., Huth, C., et al. (2002) Determinants and Consequences of Atrial Fibrosis in Patients Undergoing Open Heart Surgery. Cardiovascular Research, 54, 390-396. https://doi.org/10.1016/S0008-6363(02)00251-1

[11] Kowey, P.R., Stebbins, D., Igidbashian, L., Goldman, S.M., Sutter, F.P., Rials, S.J., et 
al. (2001) Clinical Outcome of Patients Who Develop PAF after CABG Surgery. Pacing and Clinical Electrophysiology, 24, 191-193. https://doi.org/10.1046/j.1460-9592.2001.00191.x

[12] Megens, M.R., Churilov, L. and Thijs, V. (2017) New-Onset Atrial Fibrillation after Coronary Artery Bypass Graft and Long-Term Risk of Stroke: A Meta-Analysis. Journal of the American Heart Association, 6, e007558. https://doi.org/10.1161/JAHA.117.007558

[13] Saxena, A., Dinh, D.T., Smith, J.A., Shardey, G.C., Reid, C.M. and Newcomb, A.E. (2012) Usefulness of Postoperative Atrial Fibrillation as an Independent Predictor for Worse Early and Late Outcomes after Isolated Coronary Artery Bypass Grafting (Multicenter Australian Study of 19, 497 Patients). American Journal of Cardiology, 109, 219-225. https://doi.org/10.1016/j.amjcard.2011.08.033

[14] World Health Organization (2016) International Statistical Classification of Diseases and Related Health Problems 10th Revision. http://apps.who.int/classifications/icd10/browse/2016/en

[15] Tchervenkov, C.I., Wynands, J.E., Symes, J.F., Malcolm, I.D., Dobell, A.R. and Morin, J.E. (1983) Electrical Behavior of the Heart Following High-Potassium Cardioplegia. The Annals of Thoracic Surgery, 36, 314-349. https://doi.org/10.1016/S0003-4975(10)60134-8

[16] Arsenault, K.A.Y., Crystal, E., Healey, J.S., Morillo, C.A., Nair, G.M. and Whitlock, R.P. (2013) Interventions for Preventing Post-Operative Atrial Fibrillation in Patients Undergoing Heart Surgery. Cochrane Databases of Systematic Reviews, No. 1, CD003611. https://doi.org/10.1002/14651858.CD003611.pub3

[17] Ahlsson, A., Fengsrud, E., Bodin, L. and Englund, A. (2010) Postoperative Atrial Fibrillation in Patients Undergoing Aortocoronary Bypass Surgery Carries an Eightfold Risk of Future Atrial Fibrillation and a Doubled Cardiovascular Mortality. European Journal of Cardio- Thoracic Surgery, 37, 1353-1359. https://doi.org/10.1016/j.ejcts.2009.12.033

[18] Melduni, R.M., Schaff, H.V., Bailey, K.R., Cha, S.S., Ammash, N.M., Seward, J.B., et al. (2015) Implications of New-Onset Atrial Fibrillation after Cardiac Surgery on Long-Term Prognosis: A Community-Based Study. American Heart Journal, 170, 659-668. https://doi.org/10.1016/j.ahj.2015.06.015

[19] Phan, K., Ha, H.S., Phan, S., Medi, C., Thomas, S.P. and Yan, T.D. (2015) New-Onset Atrial Fibrillation Following Coronary Bypass Surgery Predicts Long-Term Mortality: A Systematic Review and Meta-Analysis. European Journal of Cardio- Thoracic Surgery, 48, 817-824. https://doi.org/10.1093/ejcts/ezu551

[20] Villareal, R.P., Hariharan, R., Liu, B.C., Kar, B., Lee, V.V., Elayda, M., et al. (2004) Postoperative Atrial Fibrillation and Mortality after Coronary Artery Bypass Surgery. Journal of the American College of Cardiology, 43, 742-748. https://doi.org/10.1016/j.jacc.2003.11.023

[21] Schwann, T.A., Al-Shaar, L., Engoren, M.C., Bonnell, M.R., Goodwin, M., Schwann, A.N., et al. (2018) Effect of New-Onset Atrial Fibrillation on Cause-Specific Late Mortality after Coronary Artery Bypass Grafting Surgery. European Journal of Cardio-Thoracic Surgery, 54, 294-301. https://doi.org/10.1093/ejcts/ezy028

[22] Kim, M.H., Deeb, G.M., Morady, F., Bruckman, D., Hallock, L.R., Smith, K.A., et al. (2001) Effect of Postoperative Atrial Fibrillation on Length of Stay after Cardiac Surgery: The Postoperative Atrial Fibrillation in Cardiac Surgery Study [PACS(2)]. American Journal of Cardiology, 87, 881-885.

https://doi.org/10.1016/S0002-9149(00)01530-7 
[23] Almassi, G.H., Schowalter, T., Nicolosi, A.C., Aggarwal, A., Moritz, T.E., Henderson, W.G., et al. (1997) Atrial Fibrillation after Cardiac Surgery: A Major Morbid Event? Annals of Surgery, 226, 501-511.

https://doi.org/10.1097/00000658-199710000-00011

[24] Aranki, S.F., Shaw, D.P., Adams, D.H., Rizzo, R.J., Couper, G.S., VanderVliet, M., et al. (1996) Predictors of Atrial Fibrillation after Coronary Artery Surgery. Current Trends and Impact on Hospital Resources. Circulation, 94, 390-397. https://doi.org/10.1161/01.CIR.94.3.390

[25] Burgess, D.C., Kilborn, M.J. and Keech, A.C. (2006) Interventions for Prevention of Post-Operative Atrial Fibrillation and Its Complications after Cardiac Surgery: A Meta-Analysis. European Heart Journal, 27, 2846-2857. https://doi.org/10.1093/eurheartj/ehl272

[26] Zebis, L.R., Christensen, T.D., Thomsen, H.F., Mikkelsen, M.M., Folkersen, L., Sorensen, H.T., et al. (2007) Practical Regimen for Amiodarone Use in Preventing Postoperative Atrial Fibrillation. The Annals of Thoracic Surgery, 83, 1326-1331. https://doi.org/10.1016/j.athoracsur.2006.09.096

[27] Munkholm, S.B., Mortensen, P.E., Lundbye-Christensen, S. and Andreasen, J.J. (2015) Validation of Post-Operative Atrial Fibrillation in the Western Denmark Heart Registry. Danish Medical Journal, 62, A5162. 